\title{
FABRICATION OF NONRECIPROCAL MICROWAVE COMPONENTS USING THICK FILM FERRIMAGNETIC PASTES
}

\author{
CHINMAY. K. MAITI, D. BHATTACHARYYA and N. B. CHAKRABARTI \\ Indian Institute of Technology, Kharagpur 721302, India
}

\begin{abstract}
This paper reports results of investigations on edge guided mode circulators and junction circulators using thick film ferrimagnetic pastes in the frequency range from 2 to $12 \mathrm{GHz}$. Performance of the circulators realized with the thick film ferrimagnetic pastes will be compared with those made on YIG substrates in respect of insertion loss, isolation and bandwidth. The study shows that while good isolation bandwidth is obtainable with edge guided mode circulators, the insertion loss remains high.
\end{abstract}

\section{INTRODUCTION}

Low cost high reliability miniature microwave components are demanded by present day complex microwave systems. Among the various components, nonreciprocal components find extensive use in the form of isolators and circulators. In an earlier paper, ${ }^{1}$ the performance of isolators working on edge guided mode phenomena has been discussed; the component was fabricated using thick film technique on ferrimagnetic pastes developed by the authors. The method enjoys the advantage that nonreciprocal action can be obtained by screen printing a portion of alumina substrate with ferrimagnetic paste and by locally applying a magnetic field. The same technique has been utilized in fabricating circulators and the performance characteristics of such circulators have been reported in this paper. The potential for usefulness of such components in the microwave frequency range has been tested in respect of insertion loss, isolation and return loss and has been compared with the devices made on YIG substrates. The broad band characteristic of the edge guided circulators has also been established in comparison with the junction circulators.

\section{FABRICATION OF PASTE}

Presintered ferrite powders were wet milled after adding ethyl cellulose (as resin), terpineol and isobutyl alcohol (as organic vehicle) and glass powder (as binder to alumina substrate, Transene Glass Composition: TGC 130) for about 40 hours in a stainless steel pot.
The pastes so made were found to be screen printable with 200 mesh stainless steel or polyester screens with an emulsion backing of 1-4 mils. Printed films were allowed to settle for about 15 minutes and dried at about $125^{\circ} \mathrm{C}$ for 20 minutes. The optimum firing temperature is around $950^{\circ} \mathrm{C}$ with $10-15$ minutes soaking time on a belt furnace with normal air circulation. This fully destroys the residual organic matter present and matures the glass flux in the coating. Thicker films were obtained by multiple firing. Prints were thoroughly dried between printing. The quality of adhesion of the ferrimagnetic paste to the alumina substrate is found to be dependent on the amount of glass added and also varies with ferrite composition. It has been found that addition of at least $5 \%$ by weight of glass is necessary for good adhesion to the alumina substrates for Lithium ferrites.

TABLE I

\begin{tabular}{lccl}
\hline & L1250/2 & TT G 113 & TT G 610 \\
\hline $4 \pi \mathrm{M}_{\mathrm{s}}$ & 1250 & 1780 & 680 \\
$\mathrm{~T}_{\mathrm{c}}^{\circ} \mathrm{C}$ & 365 & 280 & 185 \\
$\varepsilon$ & 16 & 15 & 14.5 \\
$\tan \delta_{\mathrm{e}}$ & .0005 & .0002 & .0002 \\
$\operatorname{line}$ width $\Delta \mathrm{HG}$ & 330 & 45 & 40 \\
\hline
\end{tabular}

The properties and compositions of the ferrite paste and substrates used for thick film deposition are shown in Table I. 


\section{FABRICATION OF DEVICES AND THEIR PERFORMANCE}

The edge guided wave $e^{2,3}$ circulator using microstrip version of field displacement phenomenon in ferrite filled waveguide has been described by de Santis. ${ }^{4,5}$ This is based on the fact that edge guided waves circulate along the periphery of a metal film deposited on a ferrimagnetic substrate transversely magnetized.

Edge guided wave isolators have been reported earlier. ${ }^{1}$ The circulator described in this communication may be considered to be a combination of three isolators, with resistively loaded part placed in the common central region. The dimensions of the guiding structure have been selected with respect to the performance obtained with the isolators using ferrite pastes.

Figure 1 is a photograph of the rf circuit made by using thick film technique. The ground plane is deposited on alumina substrate with Pd-Ag conductor paste (ESL-9635) and the top conductor is made by Ag conductor paste (Transene Silver Composition type: 200). The ferrite layers are typically 6-8 mils thick, deposited on the ground plane. There are short tapering sections at the three ports for approximate matching to $50 \mathrm{ohm}$ lines.

Figure $2(\mathrm{~A}, \mathrm{~B}, \mathrm{C})$ shows the performance of such a circulator over the frequency range from $3.5-12 \mathrm{GHz}$ with an applied magnetic field $\left(H_{\mathrm{a}}\right)$ of $1250 \mathrm{G}$. The saturation magnetization for this paste is $1250 \mathrm{G}$ which corresponds to $f_{\mathrm{m}}$ equal to $3.6 \mathrm{GHz}$. For frequencies below $3.5 \mathrm{GHz}$ the insertion loss of the device exceeds $20 \mathrm{~dB}$ and is therefore excluded from measurements.

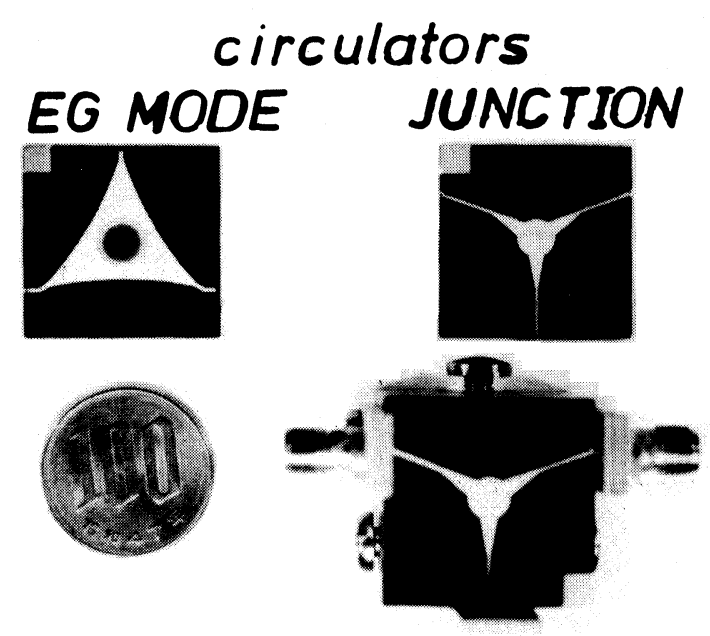

FIGURE 1 RF circuit using thick film techniques.

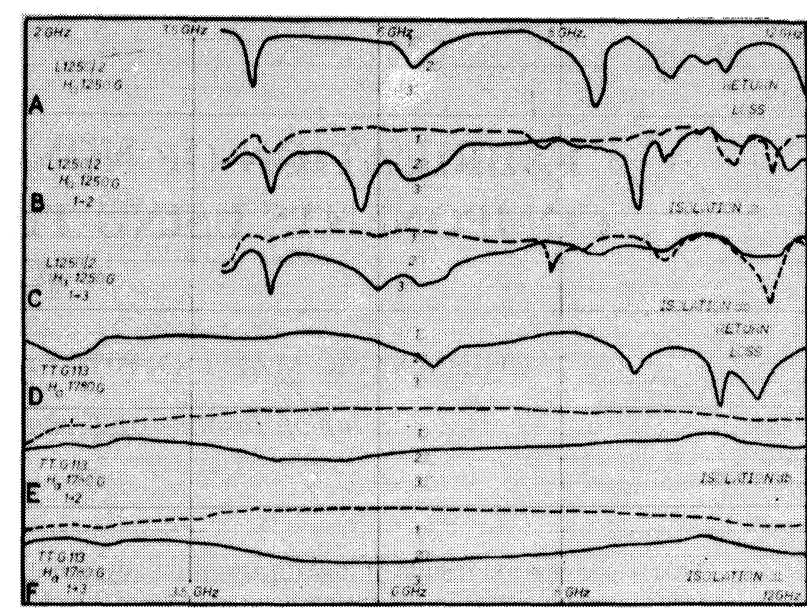

FIGURE 2 Composite diagram showing EGW circulator characteristics.

The experimental data presented here have been obtained by swept-frequency measurement using HP coax reflectometer system utilizing a dual directional coupler.

Figure $2(\mathrm{~B}, \mathrm{C})$ shows the isolation and insertion loss (broken line) for transmissions from port 1 to ports 2 and 3 respectively. It is seen that an isolation better than $20 \mathrm{~dB}$ is obtainable over a frequency range from $5-7.5 \mathrm{GHz}$. However, the insertion loss is too high and is of the order of $10 \mathrm{~dB}$. This large amount of loss is possibly due to a smaller thickness of the ferrite layer compared to 25 mil thickness of the YIG substrate and the diffusion of $\mathrm{Ag}$ in the ferrite region. The migration is accentuated because of the large porosity of printed ferrite layers.

The corresponding results for this type of circulator over YIG substrate (TT G 113) are shown in Figure 2 (D, E, F) with an applied magnetic field of $1780 \mathrm{G}$.

Figure $2(\mathrm{E}, \mathrm{F})$ shows the isolation and insertion loss (broken line) for transmissions from port 1 to ports 2 and 3 respectively. Figure 2D shows the return loss. It may be observed that an isolation of $20 \mathrm{~dB}$ is obtained over the frequency range from $4.5-8 \mathrm{GHz}$. The insertion loss is of the order of $0.5-1 \mathrm{~dB}$ throughout the useful range.

Figure $3(\mathrm{~A}, \mathrm{~B})$ shows the effect of variation of applied magnetic field. In case of YIG, with the increase of magnetic field from $1780-2800 \mathrm{G}$, the isolation exceeding $30 \mathrm{~dB}$ is obtained over the frequency range from $6.3-6.8 \mathrm{GHz}$, with an insertion loss of approximately $1 \mathrm{~dB}$. Similar increase in the peak isolation obtainable associated with a shrinkage in band width is also observed in the case of devices made on ferrimagnetic pastes. This is shown in Figure 3A. The 


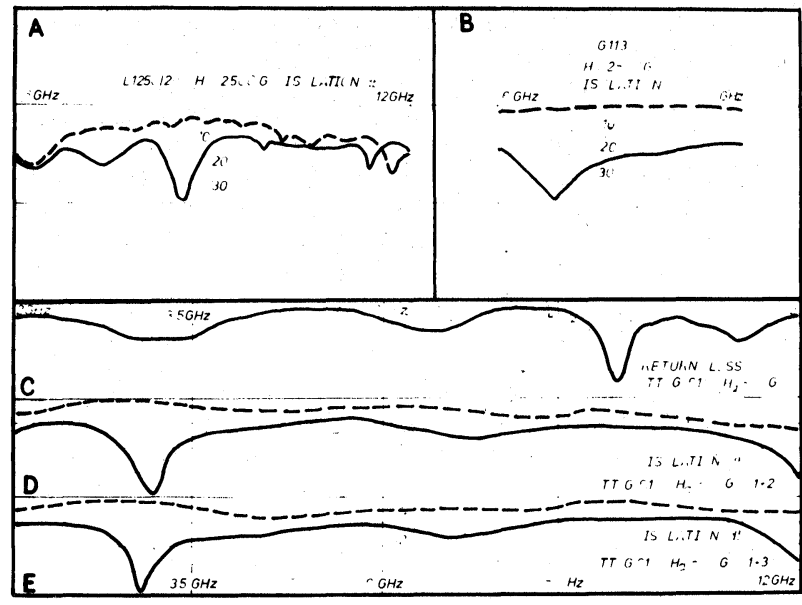

FIGURE 3 Composite diagram showing EGW circulator characteristics.

effect of variation of magnetic field, however is not examined in detail. In fact some apparent discrepancy has been observed and has to be investigated critically before any conclusion can be drawn.

Figure $3(\mathrm{C}, \mathrm{D}, \mathrm{E})$ shows the results of edge guided mode circulators on another YIG substrate (TT G $610)$. Figure $3(D, E)$ shows the isolation and insertion loss (broken line) for transmission from port 1 to ports 2 and 3 respectively. Figure $3 \mathrm{C}$ shows the return loss. It is observed that an isolation of $20 \mathrm{~dB}$ is obtained over the frequency range from $2.8-3.2 \mathrm{GHz}$. The insertion loss is approximately $1 \mathrm{~dB}$ over this range.

Three port symmetrical junction circulators have been made on both YIG substrates and on ferrimagnetic pastes developed. The disc radius is approximately $2.5 \mathrm{~mm}$ and $50 \mathrm{ohm}$ lines are matched to the disc resonator through linearly tapered transformer sections. The wave guiding structures in these devices were printed with thick film gold conductor (ESL 8880).

Figure 4 (A, B, C) shows the performance of such a circulator on ferrimagnetic films. The $20 \mathrm{~dB}$ isolation band is from $8.4-9.6 \mathrm{GHz}$ for an applied magnetic field of $2000 \mathrm{G}$. The insertion loss is about $10 \mathrm{~dB}$. The return loss is shown in Figure 4A.

The corresponding results for the junction circulator made on YIG substrate (TT G 113) are shown in Figure 4 (D, E, F). It may be seen from Figure 4 (E, F) that $20 \mathrm{~dB}$ isolation is obtained over a frequency range from $5.5-7 \mathrm{GHz}$ for transmission from port 1 to port 2 and from 5.9-6.6 for transmission from port 1 to port 3. Figure 4D shows the return loss.

The comparison of the results with those obtained in the edge guided mode circulators indicates a substantial

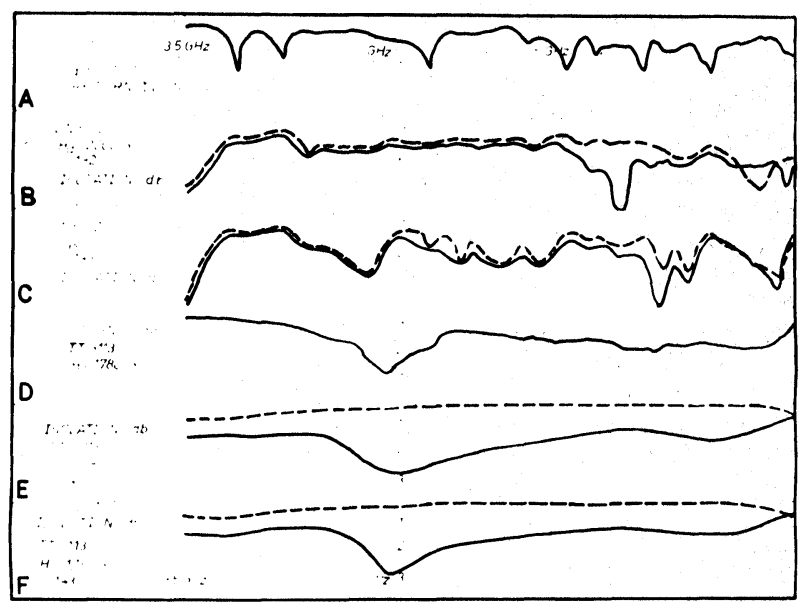

FIGURE 4 Composite diagram showing junction circulator characteristics.

improvement in bandwidth of the order of $1 \mathrm{GHz}$ for the ferrimagnetic film case and of the order of $2 \mathrm{GHz}$ for the YIG substrates.

\section{CONCLUSION}

The edge guided wave circulators using ferrimagnetic paste developed have been studied and compared with devices on YIG substrates. The principal scope of the study was to establish the feasibility of using ferrimagnetic paste in thick film fabrication of circulators and to compare bandwidth capabilities of edge guided mode circulators and junction circulators. It may be observed that $20 \mathrm{~dB}$ isolation bandwidth of edge guided mode circulators on YIG substrate and ferrimagnetic film are $3.5 \mathrm{GHz}$ and $2.5 \mathrm{GHz}$ respectively, while that for the junction circulators it is $1.2 \mathrm{GHz}$.

Unfortunately the insertion loss of ferrimagnetic pastes is rather too high. This is possibly due to smaller thickness of ferrimagnetic films and diffusion of conductor paste in ferrite zone.

The studies which are currently underway are aimed at minimizing the loss by suitable choice of ferrite-glass composition and by increasing the thickness of the ferrite layer. It should be emphasized that the designs of the devices have not been optimized. The size of the central lossy region and the geometry of the conductor pattern are expected to have considerable effect on the performance of the devices. The effect of variation of magnetic field on the performance of the circulator has not yet been properly examined; in fact there are some discrepancies in the observations. These are now being studied in detail. 


\section{ACKNOWLEDGEMENTS}

The work reported here has been supported by the Department of Science and Technology, Govt. of India, New Delhi.

Authors are grateful to Professor G. S. Sanyal for his kind permission to use the microwave measurement facilities necessary for this work and thank Mr N. C. Chakrabarti for his assistance in measurements.

\section{REFERENCES}

1. N. B. Chakrabarti C. K. Maiti, S. Kal and D. Bhattacharyya, "Microwave Applications of Ferrimagnetic
Pastes", Proc. International micro-electronic Conference, ISHM Los Angeles, 224-228 (November 1979).

2. M. E. Hines "A New Microstrip Isolator and its Application to Distributed Diode Amplification", IEEE G-MTT Int'l Microwave Symp. Digest, 304-307 (1970).

3. M. E. Hines, "Reciprocal and Nonreciprocal Modes of Propagation in Ferrite Stripline and Microstrip Devices", IEEE Trans. on Microwave Th. \& Tech., Vol: MTT-19, 442-451, No. 5 (May 1971).

4. P de Santis and F. Pucci, "Novel Type of MIC Symmetrical 3-Port Circulator", Electronics Letter, 12-13 (13th January 1972).

5. P de Santis and F. Pucci, "The Edge-Guided-Wave Circulator", IEEE Trans. on Microwave Theory \& Tech., Vol. MTT-23, 516-519, No. 6 (June 1975). 

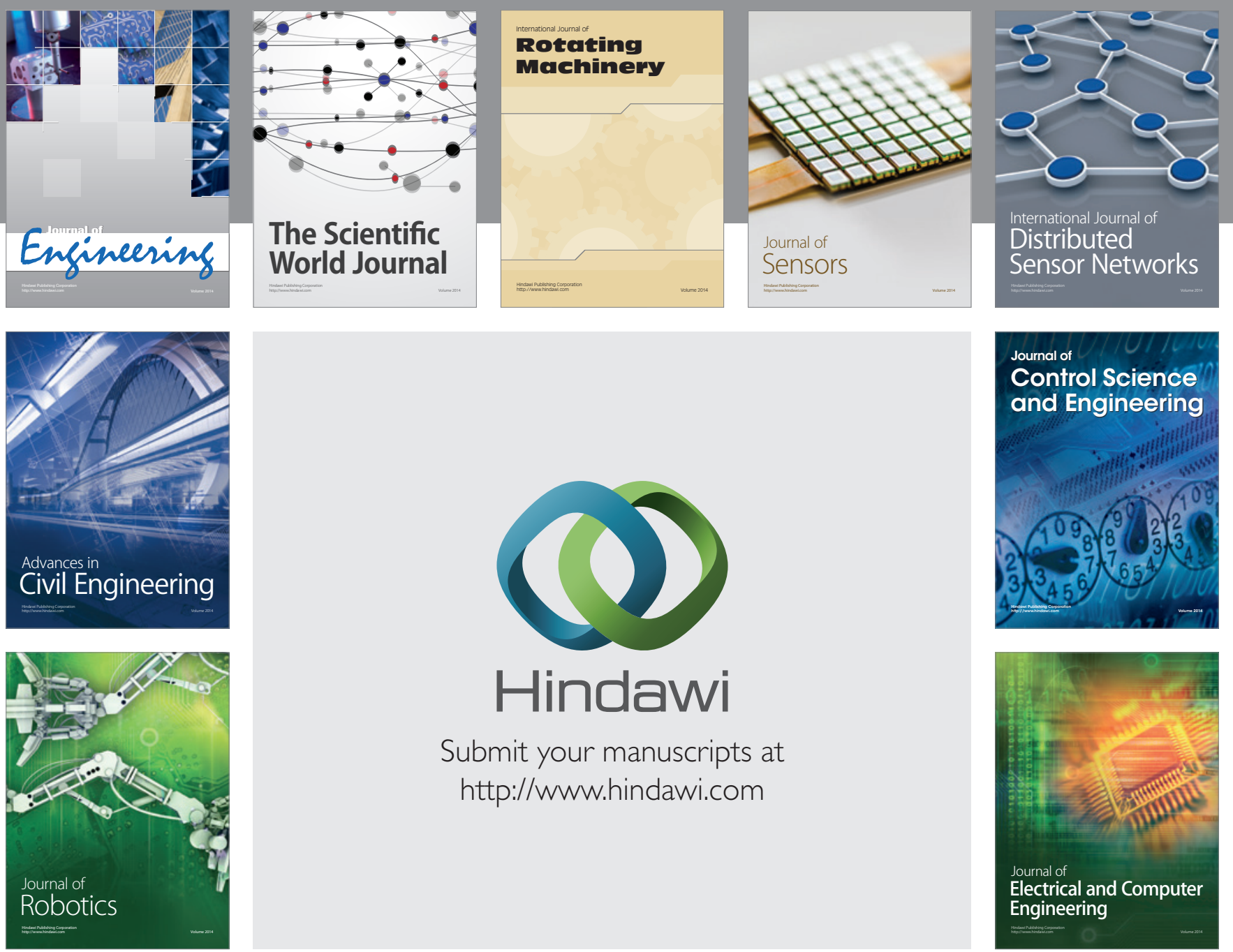

Submit your manuscripts at

http://www.hindawi.com
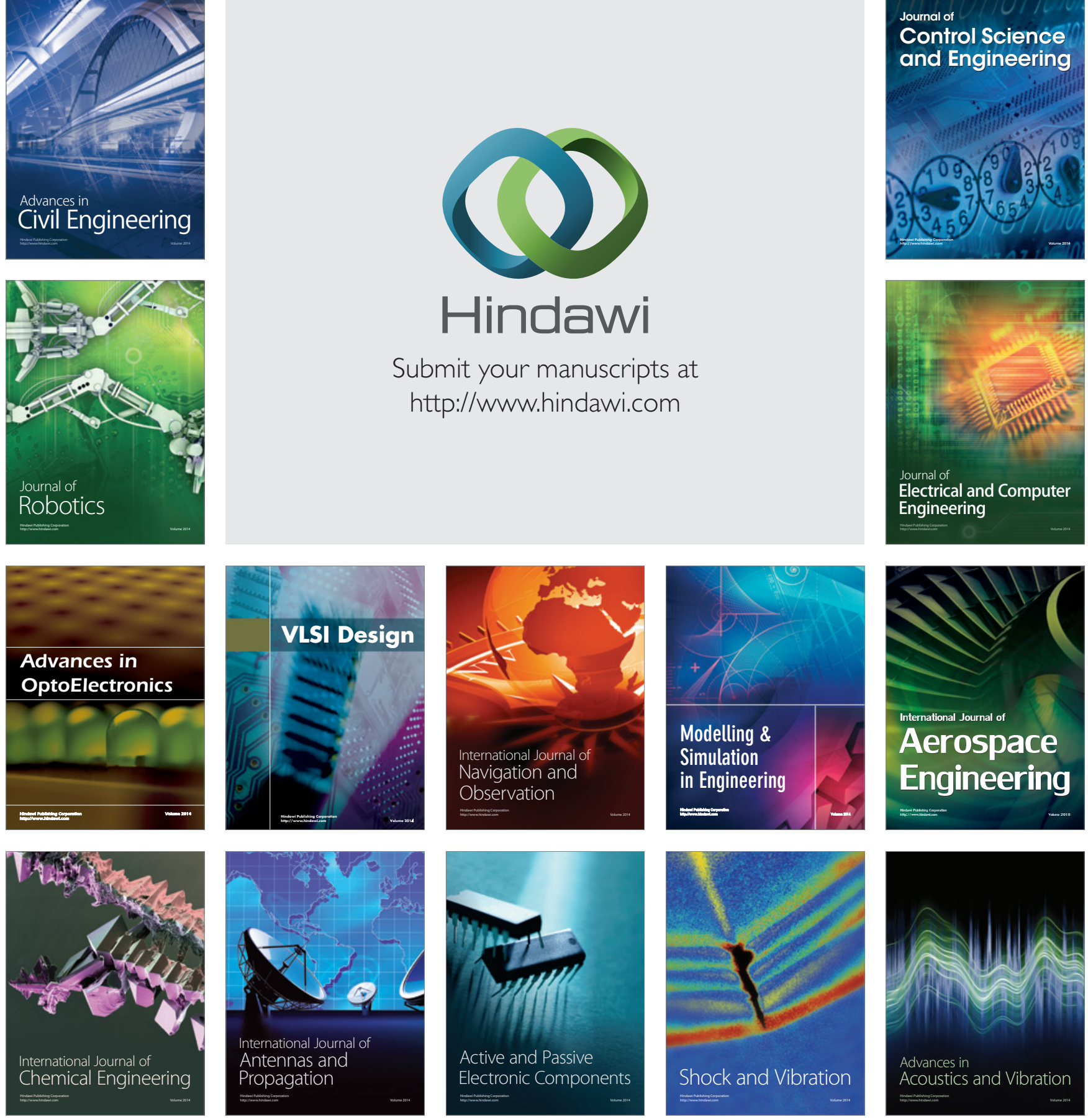